\title{
Stretchable optical fibre sensor for soft surgical robot shape reconstruction
}

\author{
Yanlin He ${ }^{1,2}$, Likun GaO ${ }^{1,2}$, Yuchen Bai ${ }^{1,2}$, Hangwei Zhu ${ }^{1,2}$, \\ GuANGKai Sun ${ }^{1,2}$, Lianqing ZhU ${ }^{1,2^{*}}$, Haidong Xu ${ }^{1}$ \\ ${ }^{1}$ Key Laboratory of the Ministry of Education \\ for Optoelectronic Measurement Technology and Instrument, \\ Beijing Information Science and Technology University, \\ Beijing, 100192, China \\ ${ }^{2}$ Beijing Laboratory of Optical Fiber Sensing and System, \\ Beijing Information Science and Technology University, \\ Beijing, 100016, China \\ *Corresponding author: zlq_2018@sina.com
}

\begin{abstract}
Soft robotics presents several advantages in the field of minimally invasive surgery. However, existing methods have not fully addressed problems related to soft robot shape sensing due to the complex motion of soft robots and the stretchable nature of the soft materials employed. This study demonstrates the shape sensing of a soft robot with a helically embedded stretchable fibre Bragg grating (FBG)-based optical fibre sensor. Unlike straight FBG embedding configurations, this unique helical configuration prevents sensor dislocation, supports material stretchability, and facilitates shape detection for various soft-robot movements. The proposed soft-robot design principle and FBG sensor are analysed and their fabrication process, which includes an FBG-written optical fibre sensor, is described. Bending experiments are conducted with the soft robot, the wavelengths of FBG sensors at different bending and telescopic movement states are obtained, and the soft-robot shape is reconstructed. Experimental results demonstrate that the maximum error between FBG sensing and the actual bending state is less than $2.5 \%$, validating the feasibility and effectiveness of the proposed helical stretchable FBG sensing method for the shape measurement of soft robots. These results indicate the potential and applicability of this shape-sensing approach in biomedical research.
\end{abstract}

Keywords: soft robotics, minimally invasive surgery, shape measurement, stretchable structure, optical fibre.

\section{Introduction}

Soft robots are made from soft, elastic materials, and are capable of mimicking the complex movements of mammals such as humans [ $\underline{1}-\underline{5}]$. These robots have attracted considerable attention in the field of minimally invasive surgery, as they offer unique opportunities in cases where conventional rigid robots are not a viable solution. In particular, soft manipulators are suitable for surgical interventions because of their small 
size, flexibility, and high dexterity in comparison to conventional minimally invasive tools $[\underline{6}-\underline{10}]$.

Considering the confined environment of minimally invasive surgery, many existing methods are unable to achieve accurate shape measurement of a soft robot, which significantly limits their practical application. Existing techniques based on conventional rigid sensors, such as encoders and strain gauges, exhibit problems related to complex cable networks, noise signal interference and electromagnetic interference. For example, magnetic navigation and positioning sensors in minimally invasive surgery have high positioning accuracies and a wide application range, however, they will produce strong magnetic field interference and can damage pacemakers and defibrillators [11-13]. Moreover, a rigid sensor will damage human tissue when the manipulator interacts with human organs. Although the accuracies of electromagnetic sensors are high, this method is not generally applicable, and the particularities of the surgical environment place significant limitations on this type of sensor. As an alternative, fibre-optic based sensors present several attractive characteristics for soft surgical robot sensing [14-21], such as small size, low weight, low loss, immunity to electromagnetic interference, and fast response. Such advantages indicate that these could be ideal sensors in biomedical and harsh surgical environments.

Optical fibre sensing has previously been investigated in relation to various types of structures $[\underline{22}, \underline{23}]$. HE et al. proposes a 3D shape reconstruction method of a soft surgical actuator that is embedded with a straight optical fibre with two FBG sensors. However, the straight FBG embedding configuration is prone to fracture and instability when the soft actuator is bent in a wide angle [22]. SEARLE et al. designed a fibre sensing system that could measure the bending state of a manipulator [23]. SiLva et al. [24] designed an optical fibre sensor that was integrated in a garment to measure elbow angles ranging from 0 to $60^{\circ}$. This system mechanically coupled optical fibres with passive cables in a basal unit that performed a sensing function. A distance-modulation array was integrated with the basal sensing unit, and the pose of the soft robot was calculated. GE et al. [25] developed a force sensor based on a fibre Bragg grating (FBG). This system consisted of an optical power meter and a laser source, and when the applied force reached $1.55 \mathrm{~N}$, there existed a proportional relationship between the optical power and force. A reference grating was also adopted to compensate for the influence of temperature. In 2016, GE et al. [26] designed a simple soft curvature sensor in which an FBG was embedded into a hyper-elastic soft silicone substrate; this curvature sensor could realise the bidirectional measurement of structures. However, considering the soft characteristics of the silicone substrate, this curvature sensor presented problems, such as low sensitivity, poor repeatability, and unstable measurements. SAREH et al. [27] designed an optical fibre sensing system based on laser power modulation, which could measure the bending angle, bending radius, and orientation of the soft manipulator. This sensing system adopted three optical fibres to interpolate the bending radius and the elongation of the soft manipulator, which undoubtedly increased the complexity of the theoretical models that described the soft manipulator. However, the 3D shape reconstruction of the motion of the soft manipulator was not 
realized. XU et al. [21] developed a force-curvature-strain model with a helically wrapped FBG for curvature, torsion, and force measurement. The validity of this design was demonstrated using two Nitinol tubes, which exhibited small deformations in comparison with soft silicone manipulators, and the effective strain measurement range of the FBG sensor was within 1\%. XU et al. [28] developed a sensing system with an FBG-written optical fibre imbedded sinusoidally in a piece of soft silicone. Experimental results demonstrated that this structure enabled $30 \%$ elongation, and thus, this optical fibre structure could be employed to implement bending and twisting measurements of soft robots in biomedical applications. Our previous studies involved the implementation of soft actuator sensing of a silicone substrate, and an FBG, which was embedded in the soft silicone substrate with a straight configuration, which could be used for the shape monitoring of a soft actuator [16-19, 22].

However, the actuation of soft robots often leads to robot elongation and continuum deformation, essentially inhibiting the use of conventional optical fibre sensors in soft robotics owing to their stiffness and lack of stretchability. But the straight FBG embedding configuration is prone to fracture and instability when the soft robots are bent in a wide angle. Consequently, this study investigates the shape measurement of soft robots with a helically embedded stretchable FBG-based optical fibre sensor. Unlike the straight FBG embedding configuration, this unique helical configuration prevents sensor dislocation, supports material stretchability, and facilitates shape detection for various soft-robot movements. The design and sensing principle of the stretchable FBG-written optical fibre sensor is described and analysed, and we experimentally investigate the performance of the proposed helical FBG configuration under conditions in which the soft robot is actuated with different bending states.

The remainder of the paper is organised as follows: the first part of Section 2 describes the general design and embedding of a stretchable helical wrapped fibre sensor in a soft silicone manipulator. The second part of Section 2 is dedicated to the sensing mechanism and modelling of stretchable helical optical fibres. The shape-measurement experimental validation of the proposed soft robot with an embedded helical configuration FBG-written optical fibre sensor is shown in Section 3. Finally, Section 4 presents the conclusions of this paper and discusses future research.

\section{Materials and methods}

\subsection{Design and fabrication of a soft surgical robot}

Figure 1 illustrates the structural design of the proposed soft surgical robot. As shown in Fig. 1a, the soft robot is composed of four pneumatic chambers used for housing the pipes for the air supply. One chamber is used for stiffening, while the other three chambers are used to implement bending and telescopic movement. The radius of the stiffening channel is $4 \mathrm{~mm}$, diameter of the pneumatic chamber is $8 \mathrm{~mm}$, length of the robot is $100 \mathrm{~mm}$, lengths of the stiffening and pneumatic chambers are $90 \mathrm{~mm}$, and length of the cap, which is fed through the pressure inlet ports to form the mechanical connection, is $10 \mathrm{~mm}$. The Kevlar fibre was wound in a double-helix pattern, and glued onto the robot 


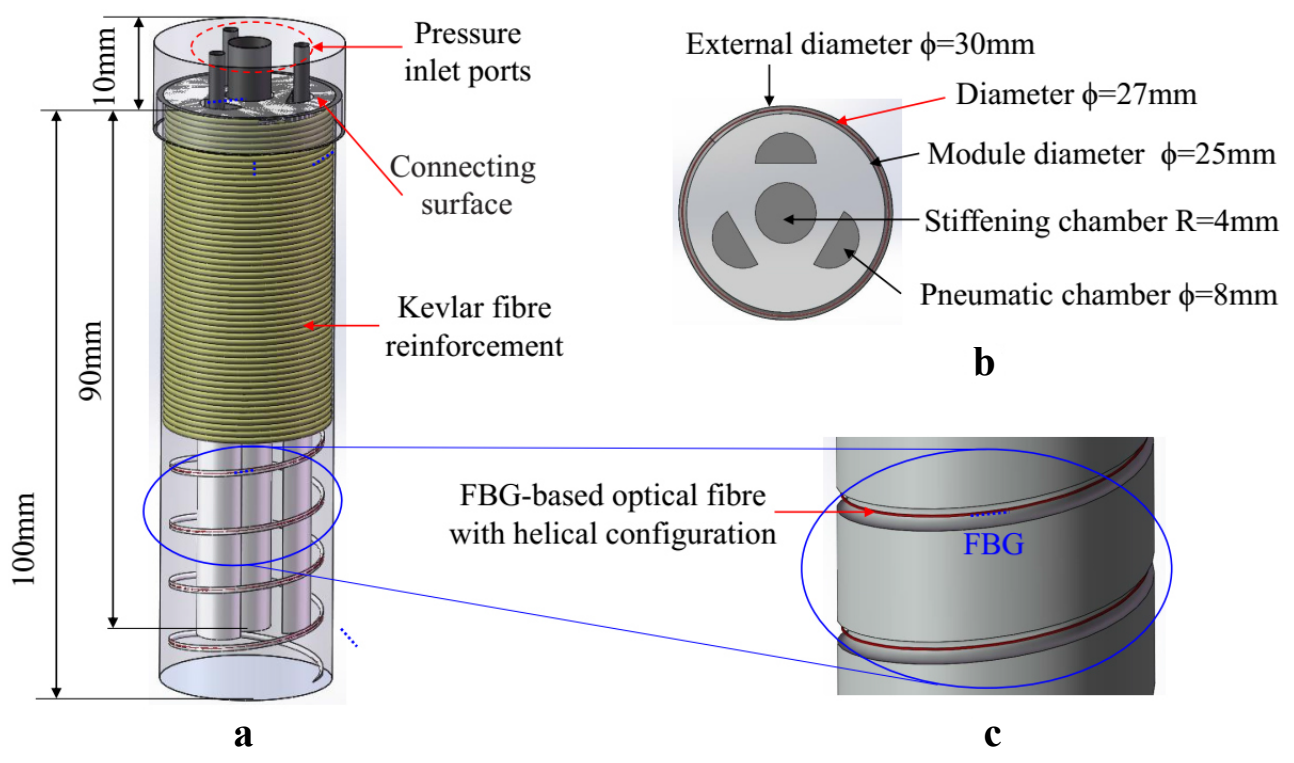

Fig. 1. Soft robot embedded with FBG-based optical fibre in a helical configuration. (a) Structure details, (b) top view of the connecting surface of the robot and cap, and (c) enlarged state of the FBG-based optical fibre.

to restrict its radial expansion. The 48-com-long FBG-written optical fibre has three FBGs and is embedded in the soft robot in a helical configuration. This unique helical configuration enables the optical fibre to be stretched and elongated with the soft robot without fracture, and with only a distortion of the helical shape and slight elongation of the fibre. Figure $1 \mathbf{b}$ depicts a detailed top view of the structure, showing the connecting surface of the robot and cap. Figure 1c depicts the enlarged state of the FBG-based optical fibre sensor. As we can see, the grating length is $10 \mathrm{~mm}$. The helical groove is designed to hold the FBG-based optical fibre in position.

To fabricate a soft robot embedded with an FBG-based helical optical fibre, we used a 3D printer (Objet Connex 500) to print our moulds, including the soft robot base mould (\#1) with a helical groove (Fig. 2a), the encapsulation mould (\#2) (Fig. 2c), the air chamber moulds, the port base (Fig. 2a), and the connector for connecting the pressure inlet ports. The entire fabrication process is illustrated in Fig. 2. The soft robot base (Ecoflex-0050 A and B, USA; see Fig. 2a) is manufactured using the helical groove (mold\#1), chamber mould, and a base mould, and curing of the silicone gel is performed at a temperature of $60^{\circ} \mathrm{C}$ for $30 \mathrm{~min}$. The air chamber mould consists of one round and three half round steel rods, the helical groove of mould \#1 is designed to embed the optical fibre in position, and the soft robot is then fabricated with a helical groove on its surface. The FBG-based optical fibre is then wound along and glued onto the helical groove of the soft robot (see Fig. 2b). The centre wavelengths of the three FBGs embedded in the soft robot are $1531.0250,1534.7563$, and $1538.6750 \mathrm{~nm}$, respec- 


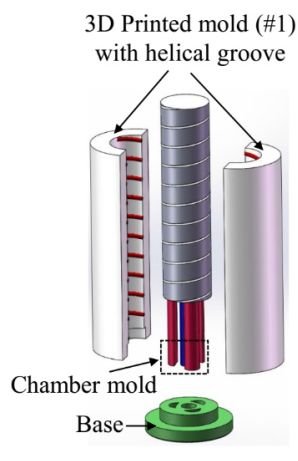

a

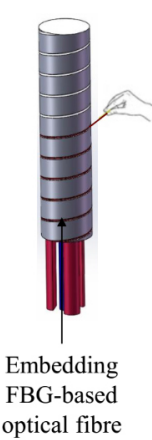

b

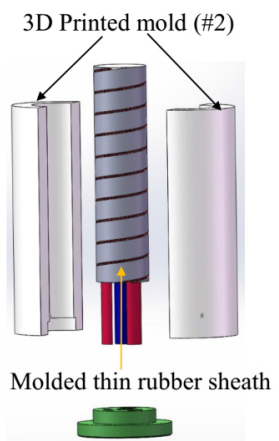

c

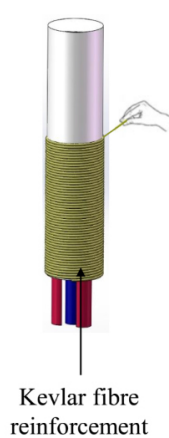

d

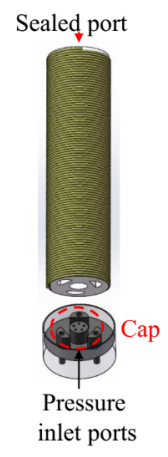

e

Fig. 2. Multistep moulding process of the soft robot with helically embedded FBGs-based optical fibre sensor. (a) First helical groove moulding step of the soft robot base with a helical groove; (b) FBG-written optical fibre is wound along the helical groove of the soft robot; (c) second moulding step: the soft robot is encapsulated in a layer of silicone to anchor the optical fibre sensor; (d) thread (Kevlar fibre) is then wound along the entire length of the robot. (e) In the final step, the base and chamber mould are removed and one end of the robot is capped, allowing for three ports for the inflow/outflow of pressure.

tively, FBG grating length is $10 \mathrm{~mm}$, bandwidth is $10 \mathrm{~dB}$, and reflectivity is approximately $70 \%$. The soft robot with embedded helical optical fibre is then encapsulated in a 1.0-mm-thick silicone layer by placing it in mold\#2 (see Fig. 2c). In addition, a single Kevlar fibre is wound around the robot in a double-helix pattern to restrict its radial expansion, and glued onto its body (see Fig. 2d). Finally, the chamber mould is removed, and a 3D-printed cap is fed through the pressure inlet ports of the robot to form the mechanical connection for the pneumatic tubes (see Fig. 2e) [르, $\underline{30}]$.

\subsection{Modelling of FBG-based optical fibre with a helical configuration}

According to the mechanisms of FBG sensors and coupling theory, when broadband light passes the FBG sensor, the wavelength of reflection can be expressed as [22]

$$
\lambda_{\mathrm{B}}=2 n_{\mathrm{eff}} \Lambda
$$

Here, $n_{\text {eff }}$ represents the effective refractive index of the guided fibre mode, and $\Lambda$ represents the period of grating.

The grating period $\Lambda$ for the FBG sensors will alter due to the changes in the strain of the grating and temperature. The relationship between the strain of the measurement point and the Bragg wavelength shift can be written as

$$
\Delta \lambda_{\mathrm{B}} / \lambda_{\mathrm{B}}=\left(1-P_{\varepsilon}\right) \varepsilon+\left(\alpha_{\mathrm{T}}+\xi\right) \Delta T
$$

Here, $\Delta \lambda_{\mathrm{B}}$ is the change in wavelength, $\varepsilon$ represents the FBG sensor strain, $P_{\varepsilon}$ is a constant value calculated from the photo-elastic coefficient of the fibre material, $\alpha_{\mathrm{T}}$ rep- 


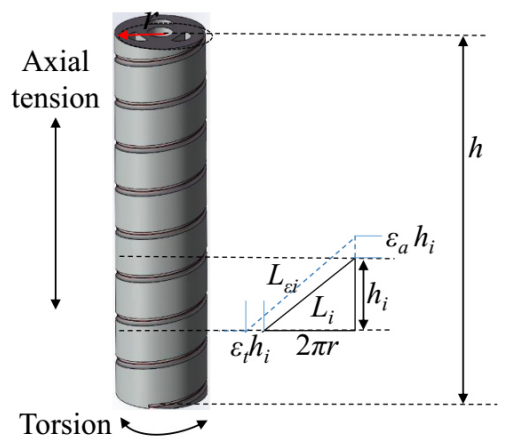

a

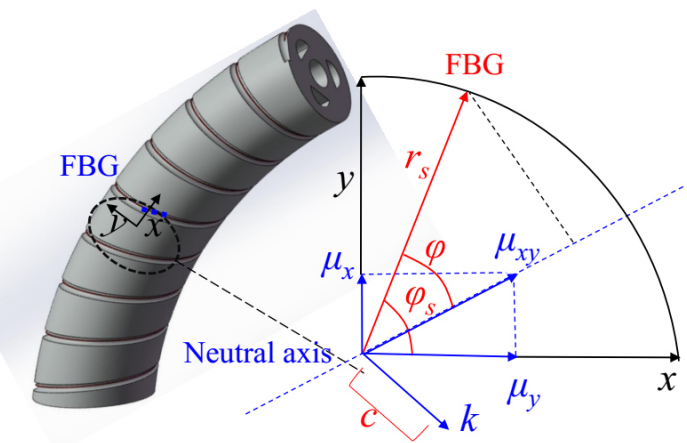

b

Fig. 3. Modelling of the soft manipulator with a helically wrapped FBG sensor. (a) Axial tension and torsion motion; (b) bending motion.

resents the expansion coefficient of the grating thermal, $\xi$ represents the thermo-optical coefficient of the grating, and $\Delta T$ represents the temperature change.

The influence of temperature on the FBG sensor is neglected because this type of soft manipulator is commonly used at room temperature with only minor variations.

$$
\Delta \lambda_{\mathrm{B}}=\lambda_{\mathrm{B}}\left(1-P_{\varepsilon}\right) \varepsilon
$$

The optical fibre sensor is fixed on the soft robot in a helical layout, and both axial and shear strain exist in the sensor. The relationship between the curvature and strain of the optical FBG sensor that is fixed on the soft manipulator is derived. A curvature-strain model describing the optical FBG sensor with a helical wrapped structure is illustrated in Fig. 3. This system is capable of assessing the bending magnitude, but not the direction. The helical optical fibre is flattened into a right triangle on a plane [21].

Suppose that the soft manipulator is divided into $i$ segments, $r$ is the radius of the soft manipulator, $h$ is the length of the manipulator, $L$ is the length of the optical fibre, $\varepsilon_{\mathrm{a}}$ is the axial strain, $\varepsilon_{\mathrm{t}}$ is the shear strain, $k$ is the curvature, $u_{x}$ and $u_{y}$ are the curvatures under deformation, and $\mu_{x y}$ is the curvature vector. Note that $\left\|\mu_{x y}\right\|=\|k\|=c, r_{\mathrm{s}}$ and $\varphi_{\mathrm{s}}$ are the position vector and orientation angle of the FBG sensor, respectively, and $\varphi$ is the angle between $r_{\mathrm{s}}$ and the neural axis. The length of the helical fibre can be calculated as:

$$
l=\sum_{i=1}^{n} l_{i}=\sum_{i=1}^{n} \sqrt{(2 \pi r)^{2}+h_{i}^{2}}
$$

Considering the breakage and bending loss, the FBG sensor is wrapped around the soft manipulator at an angle of $40^{\circ}$. When the soft manipulator exhibits tension and torsion movement, its height and width will be $\left(1+\varepsilon_{\mathrm{a}}\right) h_{i}$ and $\left(2 \pi r+\varepsilon_{\mathrm{t}} h_{i}\right)$, respectively. The resulting strain in the optical fibre and the length of the strained fibre can be expressed as: 


$$
\begin{aligned}
& \varepsilon_{l}=\left(l_{\varepsilon} / l\right)-1 \\
& l_{\varepsilon}=\sqrt{\left(2 \pi r+\varepsilon_{\mathrm{t}} h\right)^{2}+\left(1+\varepsilon_{\mathrm{a}}\right)^{2} h^{2}}
\end{aligned}
$$

Considering that the effective strain measurement range of the FBG sensor is less than $1 \%$, which means that the axial strain $\varepsilon_{\mathrm{a}}$ and shear strain $\varepsilon_{\mathrm{t}}$ are all within 0.01 , Eq. (2) can be linearized with respect to $\left(\varepsilon_{\mathrm{a}}, \varepsilon_{\mathrm{t}}\right)$ at the point $(0,0)$. The axial strain $\varepsilon_{\mathrm{a}}$ and shear strain $\varepsilon_{\mathrm{t}}$ of the soft manipulator can be expressed as:

$$
\begin{aligned}
& \varepsilon_{\mathrm{a}}=\left\|\mu_{x y} \times r_{\mathrm{s}}\right\|=\left\|\left[\begin{array}{l}
\mu_{x} \\
\mu_{y}
\end{array}\right] \times\left[\begin{array}{l}
r \cos \alpha_{\mathrm{s}} \\
r \sin \alpha_{\mathrm{s}}
\end{array}\right]\right\|=\left\|\left[\begin{array}{l}
\mu_{x} \\
\mu_{y}
\end{array}\right]\right\|\left\|\left[\begin{array}{l}
r \cos \alpha_{\mathrm{s}} \\
r \sin \alpha_{\mathrm{s}}
\end{array}\right]\right\| \sin \alpha=c r \sin \alpha \\
& \varepsilon_{\mathrm{t}}=\left(\mu_{z}-\hat{\mu}_{z}\right) r
\end{aligned}
$$

where $\hat{\mu}_{x}$ and $\hat{\mu}_{y}$ are the bending pre-curvatures and $\hat{\mu}_{z}$ is the pre-torsion within the soft manipulator. $\mu_{x}, \mu_{y}$ and $\mu_{z}$ are the curvatures and torsion under deformation. $r_{\mathrm{s}}$ and $\alpha_{\mathrm{s}}$ are the position vector and orientation angle of the FBG, $c$ is the magnitude of the bending curvature, and $\alpha$ is the angle between $r_{\mathrm{s}}$ and the neutral axis. The relationship between curvature and strain of the helically wrapped FBG sensor are obtained by Eq. (6), and the influence of temperature is not considered owing to the room temperature

$$
\varepsilon_{l} \approx \frac{2 \pi r}{l^{2}} \varepsilon_{\mathrm{a}}+\frac{h^{2}}{l^{2}} \varepsilon_{\mathrm{t}}
$$

\section{Experimental results and discussion}

To validate the proposed helical embedded sensing technology and modelling techniques described above, a series of bending experiments were conducted on the soft robot that was embedded with three FBGs in a helical configuration. Figure 4 illustrates the experimental setup, which includes the soft manipulator, pneumatic control system, FBG sensing system, and computer. The original centre wavelengths of the three FBGs (FBG1, FBG2, and FBG3) that were embedded in the soft robot in a helical configuration were 1544,1546 , and $1546 \mathrm{~nm}$, their peak reflectivities were $70 \%$, their response times were $70 \mathrm{~ms}$, the typical temperature sensitivity FBGs is $0.1^{\circ} \mathrm{C}$, and a smoothing denoising algorithm could also be adopted to let the FBG capable to resolve variations of $<0.1{ }^{\circ} \mathrm{C}$. The input pressure of the soft robot was controlled by the pneumatic control system, an AIRTAC VALVE model was used to input pressure in the experiment, and the received optical signals were translated into digital signals and extracted by the computer. A broadband light source with wavelengths from 1529 to $1605 \mathrm{~nm}$ was launched in the FBG as input light, and measured by an optical spectrum analyser (OSA) with a resolution of $0.5 \mathrm{pm}$. An AQ6370C spectral analyser (Yokogawa Corporation, Japan) was 


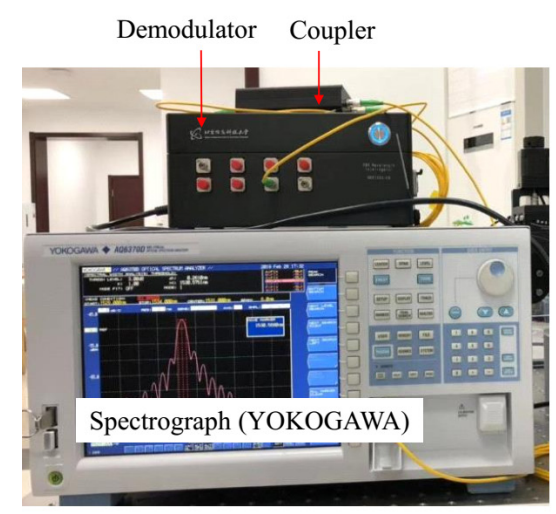

FBG sensing system

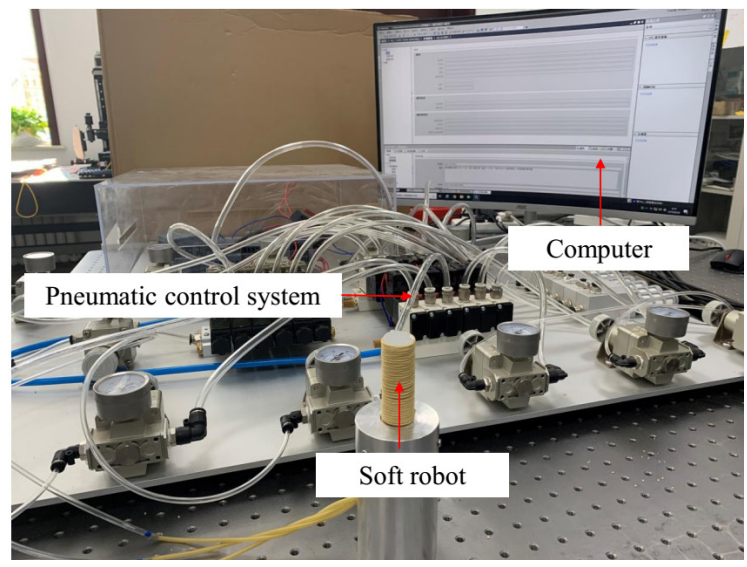

Fig. 4. Experimental setup for measuring compression and bending movements.

used as the spectrograph to reflect the FBG signal, which had a wavelength measurement range of 600-1700 $\mathrm{nm}$ and a power range of +20 to $-90 \mathrm{dBm}$. The demodulator used in this work was developed by our team, and provided a wavelength range of 1525-1610 nm, and a demodulation rate of $35 \mathrm{kHz}$. A LabVIEW program was developed to store the data and reconstruct the bending shape of the soft robot.

The elongation of the soft robot was obtained from three simultaneously activated pneumatic chambers. Images of the elongation movement of the soft robot are shown in Fig. 5. When the activation pressure of each chamber was 0.65 bar, the soft robot began to extend from its initial length of $100 \mathrm{~mm}$ to a final length of $156.2 \mathrm{~mm}$, corresponding to an elongation of $56.2 \%$. Figure 6 shows the experimental results for the soft robot with elongation. The relationship between pressure and length follows the same trend as in the elongation movement, and the Kevlar fibre restricts the radial expansion of the soft robot, ensuring that it only stretches to within a safe working range.

When the soft robot was actuated by the pneumatic control system with various pressures, the soft robot bent to different angles between 0 and $160^{\circ}$, as shown in the move-
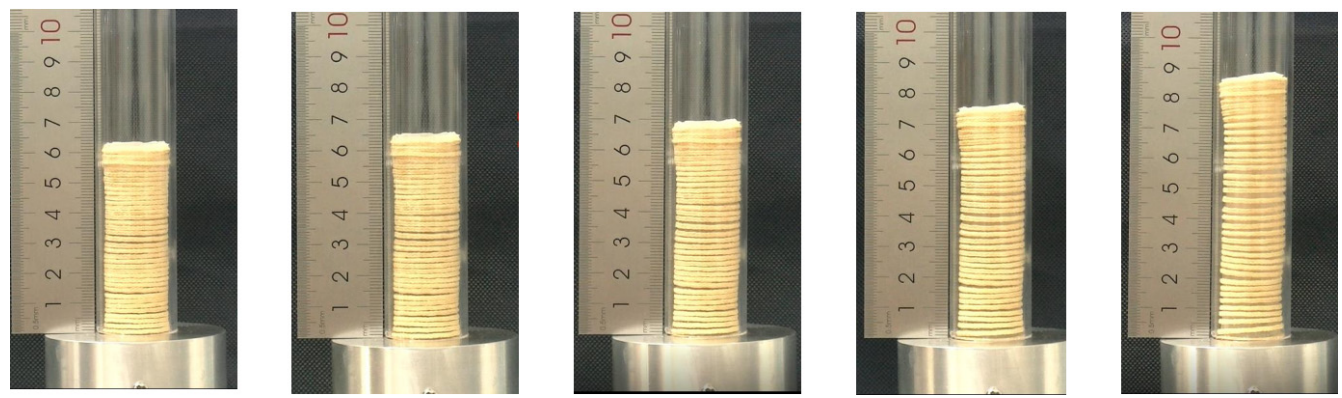

Fig. 5. Experimental snapshots of the soft robot during elongation movement. 


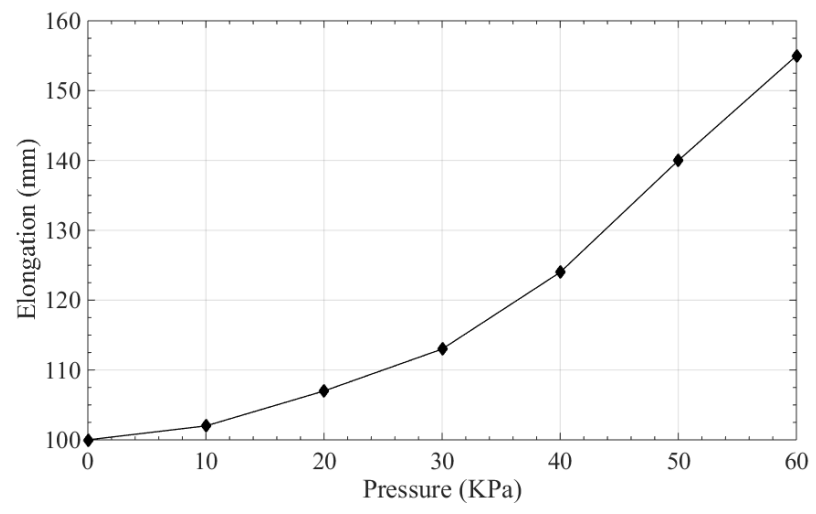

Fig. 6. Experimental results for the elongation movement of the soft robot.

ment snapshots depicted in Fig. 7. Figure 8 shows that the bending angle of the soft robot increases with increasing input pressure. When the input pressure is less than $25 \mathrm{kPa}$, it is free to expand in any direction owing to the expansion of each chamber, and thus, the bending angle of the soft robot increases gradually. When the input pressure reaches
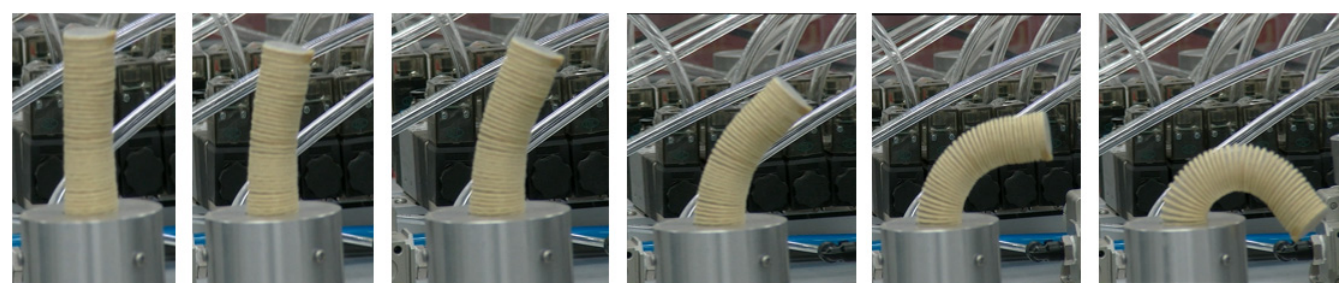

Fig. 7. Bending experiment snapshots of the soft robot.

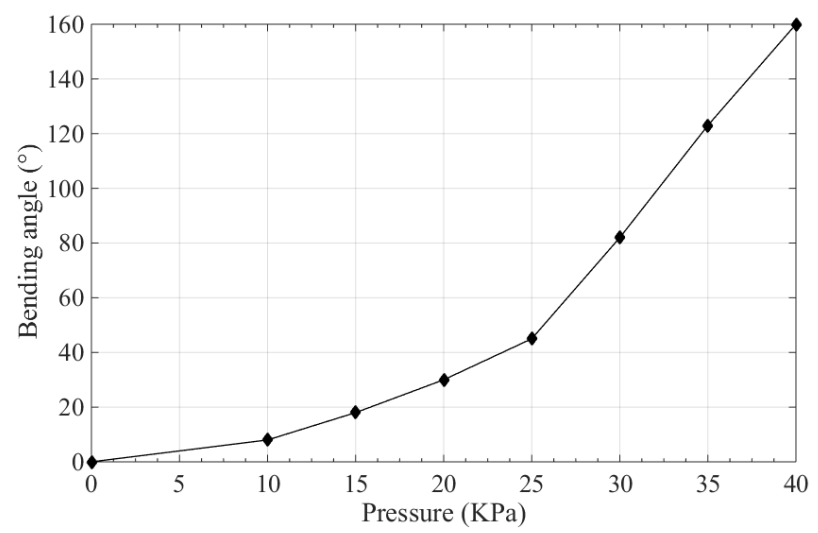

Fig. 8. Experimental results for the soft robot with bending and elongation movement. 

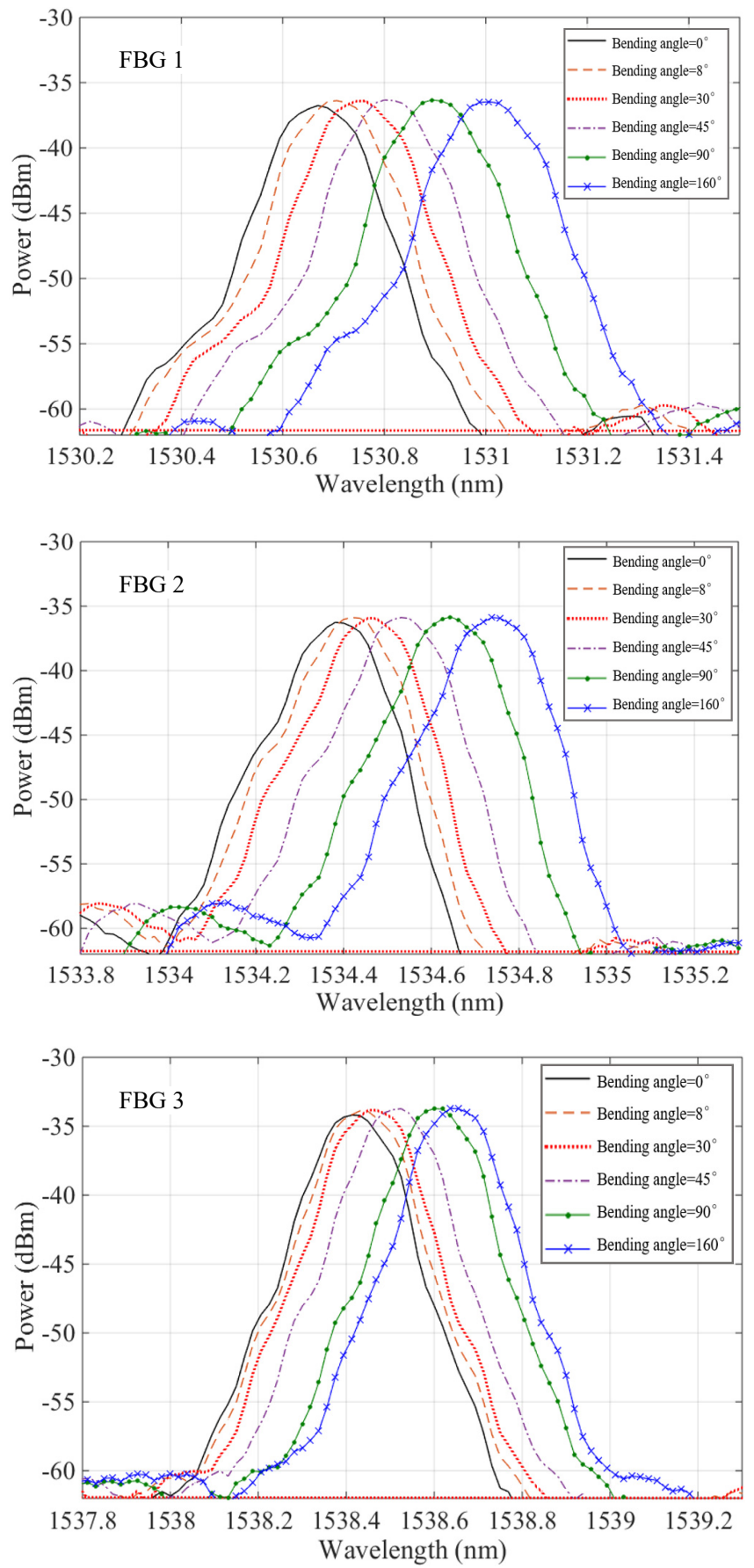

Fig. 9. Wavelength shifts of the three FBG sensors at different bending states. 
$0.025 \mathrm{MPa}$, the interaction between the chamber and the Kevlar fibre begins to limit the radial expansion, thus leading to a considerable reduction improvement in bending.

The wavelength and power of the three FBG sensors when the soft robot bending angles are $0^{\circ}, 8^{\circ}, 30^{\circ}, 45^{\circ}, 90^{\circ}$, and $160^{\circ}$, respectively, are depicted in Fig. 9. As shown in Fig. 9, when the soft robot bending angle ranges between 0 and $80^{\circ}$, the power peaks of FBG 1 increase from 1530.7063 to $1531.0062 \mathrm{~nm}$, those of FBG 2 range from 1534.3812 to $1534.7375 \mathrm{~nm}$, and those of FBG 3 range from 1538.4125 to $1538.6375 \mathrm{~nm}$. The FBG sensor wavelengths gradually increased with increasing bending angle, the reflected peak wavelengths of the FBG sensors gradually shifted towards larger wavelengths, and the wavelength shifts of the FBGs were $0.3374,0.3563$, and $0.225 \mathrm{~nm}$ for FBG 1, FBG 2, and FBG 3, respectively.

The relationship between the wavelength shift and the soft robot bending angle is shown in Fig. 10, indicating that the wavelength shifts of FBG sensors increase with increasing bending angle. As we can see in the figure, owing to the combination of the flexible soft robot with a low Young's modulus and the rigid optical fibre sensor with a high Young's modulus, there are some problems, such as creep caused by rigid flexible strain coupling, and the relationship between the wavelength shift and bending angle is not linear. During the experiment, we found that the hysteresis of our sensor was approximately $1 \mathrm{~min}$. Although there exists the hysteresis of helical stretchable FBG sensor, the helical stretchable FBG sensing method for the measurement of soft robots is feasible and effective.

The 3D shape of the soft robot is reconstructed using the interpolation and curve fitting function, and the reconstructed shapes of the soft robot at different bending angles are depicted in Fig. 11. Comparing the images generated by FBG sensing and experiments in Figs. 7 and 11, we find that the bending profiles of the soft robot are the same, and the shape of the soft actuator is well reconstructed.

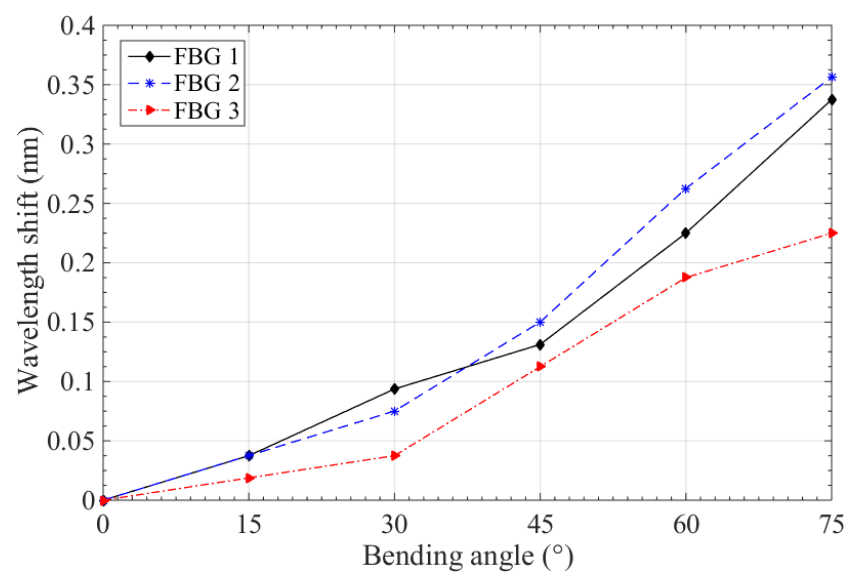

Fig. 10. Wavelength shifts of the three FBG sensors under different curvatures. 

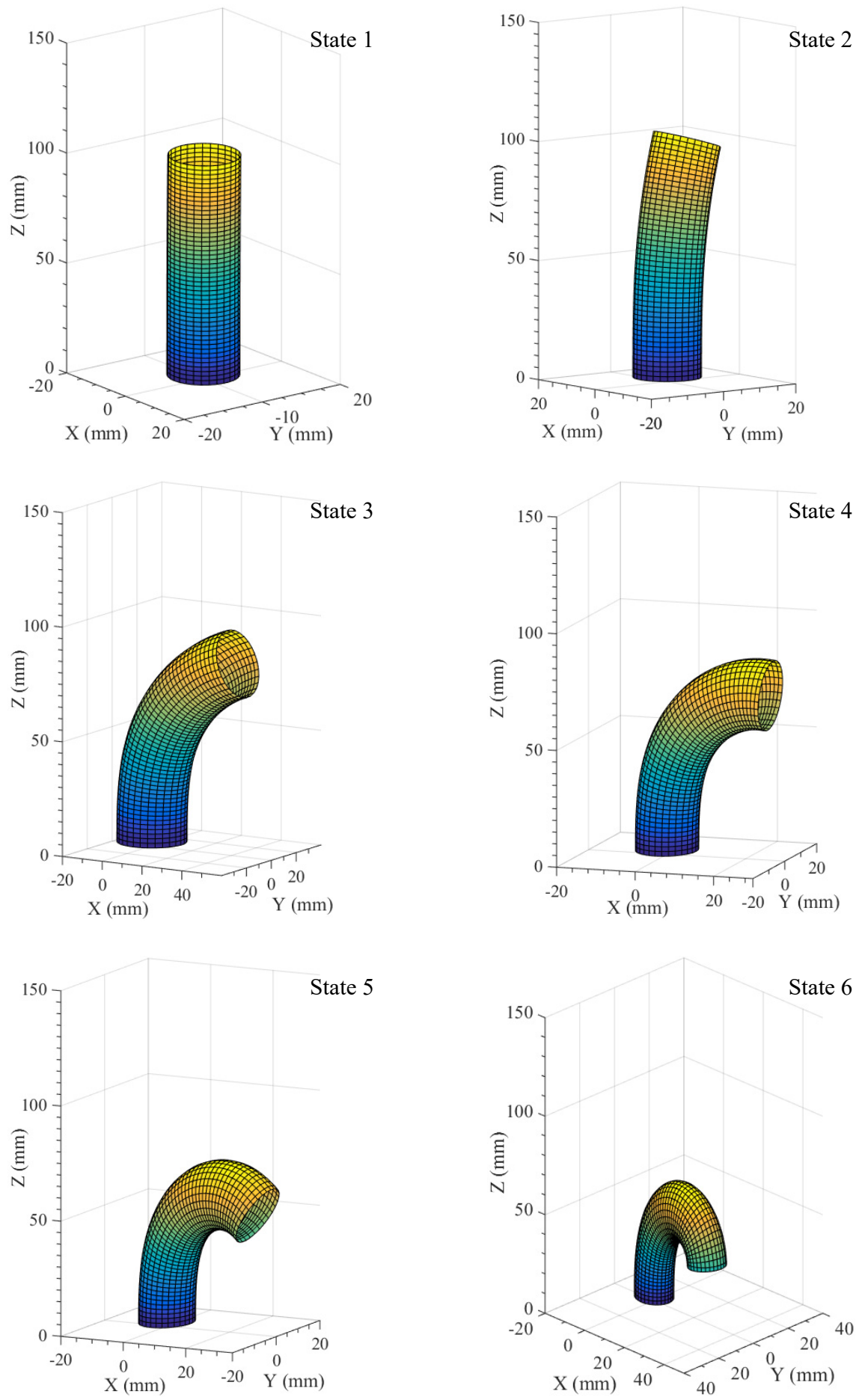

Fig. 11. Shape reconstruction of the soft actuator at different bending states. 
$\mathrm{T}$ a b 1 e. Experimental results for bending angle and errors.

\begin{tabular}{llllll}
\hline FBG sensing angle [deg] & 8.2 & 30.5 & 44.6 & 91.6 & 161.9 \\
Actual bending angle [deg] & 8 & 30 & 45 & 90 & 160 \\
Relative error [\%] & 2.50 & 1.67 & 0.89 & 1.78 & 1.19 \\
\hline
\end{tabular}

The Table presents the error between the actual bending angle of the soft robot and the FBG sensing results. As we can see, the maximum error between the FBG sensing results and the actual angle is less than $2.5 \%$. This highlights the feasibility and effectiveness of the proposed shape-reconstruction method. The responses of the three FBGs that were embedded in the soft robot in a helical configuration were highly sensitive, and the bending states of the robot were accurately reconstructed and measured.

Researchers have exerted tremendous efforts to implement the shape sensing capability of soft robots. Fibre-optic-based sensors have become increasingly popular in soft surgical robot sensing. Silva et al. [24] designed an optical fibre sensor that was integrated into a garment to measure elbow angles ranging from 0 to $60^{\circ}$, but its measurement angle range cannot meet the requirements of large-scale elongation and continuum bending deformation of soft robots. GE et al. [26] designed a simple soft curvature sensor with an FBG embedded into a hyper-elastic soft silicone substrate, which could realise the bidirectional measurement of structures. SAREH et al. [27] designed an optical fibre sensing system that could measure the bending angle, bending radius, and orientation of a soft manipulator. XU et al. [208] developed a sensing system with an FBG-written optical fibre imbedded sinusoidally in a piece of soft silicone, which enabled $30 \%$ elongation, but the 3D shape reconstruction of the motion of the soft manipulator was not realized. Our previous studies involved the implementation of soft actuator sensing of a silicone substrate, as well as an FBG, which was embedded into the soft silicone substrate with a straight configuration and could be used for the shape monitoring of a soft actuator [22]. However, the straight FBG embedding configuration is prone to fracture and instability when the soft actuator is bent in a wide angle. Although some studies have been conducted previously on curvature sensing of soft robots, few of them have involved quantitative analysis of the actuation of soft robots, which often leads to robot elongation and continuum deformation, essentially inhibiting the use of conventional optical fibre sensors in soft robotics, owing to their stiffness and lack of stretchability. The straight FBG embedding configuration is prone to fracture and instability when the soft robots are bent in a wide angle, this study investigates the shape measurement of soft robots with a helically embedded stretchable FBG-based optical fibre sensor. And unlike the straight FBG embedding configuration, this unique helical configuration prevents sensor dislocation, supports material stretchability, and facilitates shape detection for various soft-robot movements.

\section{Conclusions and future work}

A simple and effective FBG-based optical fibre sensor would certainly be advantageous for the shape sensing of soft robots. In this study, we investigated the shape measure- 
ment of soft robots with a helically embedded stretchable FBG-based optical fibre sensor, and we described and analysed the design and sensing mechanism of the sensor. The experimental results show that upon applying an activation pressure of 0.65 bar in each chamber, the soft robot could extend from its initial length of $100 \mathrm{~mm}$ to a final length of $156.2 \mathrm{~mm}$, corresponding to an elongation of $56.2 \%$. When the soft robot bending angles ranged from 0 to $160^{\circ}$, the three FBGs that were embedded in the soft robot in a helical configuration were highly sensitive and responsive, and the bending states of the robot were accurately reconstructed and measured. The experiments and data validated the feasibility and effectiveness of the proposed shape-reconstruction method. To the best of our knowledge, few investigations have involved quantitative analysis of the shape sensing of soft surgical robots with helically embedded stretchable FBG-based optical fibre sensors.

This study demonstrated a shape-sensing method for soft robots with a helically embedded stretchable FBG-based optical fibre sensor. In contrast to straight FBG embedding configurations, the helical configuration employed prevents sensor dislocation, supports stretchability, and facilitates robot movement. The experimental results obtained using our fabricated soft robot demonstrate a maximum error between the FBG sensor data and actual robot bending angle of less than $2.5 \%$. This performance indicates the feasibility and effectiveness of this approach in measuring the shape of soft robots embedded with FBG-based optical fibres in a helical configuration. This method has the potential for use in shape and pose sensing in biomedical applications, such as minimally invasive surgery.

Acknowledgment - This work is supported by the National Natural Science Foundation of China (61903041). This work is also supported by the Beijing Natural Science Foundation of China (7202017, 4204101), the Research Project of Beijing Municipal Natural Science Foundation \& Beijing Education Committee (KZ201911232044, KM201911232005); by The National Key Research and Development Project (2020YFA0711200), by the Beijing Nova Program of Science and Technology under grant No. Z191100001119052.

\section{References}

[1] Suzumori K., Iikura S., Tanaka H., Applying a flexible microactuator to robotic mechanisms, IEEE Control Systems Magazine 12(1), 1992, pp. 21-27, DOI: 10.1109/37.120448.

[2] Piccigallo M., Scarfogliero U., Quaglia C., Petroni G., Valdastri P., Menciassi A., Dario P., Design of a novel bimanual robotic system for single-port laparoscopy, IEEE/ASME Transactions on Mechatronics 15(6), 2010, pp. 871-878, DOI: 10.1109/TMECH.2010.2078512.

[3] Martinez R.V., Branch J.L., Fish C.R., Jin L., Shepherd R.F., Nunes R.M.D., Suo Z., Whitesides G.M., Robotic tentacles with three-dimensional mobility based on flexible elastomers, Advanced Materials 25(2), 2013, pp. 205-212, DOI: 10.1002/adma.201203002.

[4] Gong Z., Cheng J., Chen X., Sun W., Fang X., Hu K., Xie Z., Wang T., Wen L., A bio-inspired soft robotic arm: kinematic modeling and, hydrodynamic experiments, Journal of Bionic Engineering 15, 2018, pp. 204-219, DOI: $10.1007 / \mathrm{s} 42235-018-0016-\mathrm{x}$.

[5] Luo M., Skorina E.H., Tao W., Chen F., Ozel S., Sun Y., Onal C.D., Toward modular soft robotics: proprioceptive curvature sensing and sliding-mode control of soft bidirectional bending modules, Soft Robotics 4(2), 2017, pp. 117-125, DOI: 10.1089/soro.2016.0041. 
[6] Zolfagharian A., Kouzani A.Z., Khoo S.Y., Moghadamet A.A., Gibson I., Kaynaka A., Evolution of $3 D$ printed soft actuators, Sensors and Actuators A: Physical 250, 2016, pp. 258-272, DOI: $10.1016 /$ j.sna.2016.09.028.

[7] Ranzani T., Gerboni G., Cianchetti M., Menciassi A., A bioinspired soft manipulator for minimally invasive surgery, Bioinspiration \& Biomimetics 10(3), 2015, article 035008, DOI: $10.1088 /$ $1748-3190 / 10 / 3 / 035008$.

[8] Polygerinos P., Wang Z., Galloway K.C., Wood R.J., Walsh C.J., Soft robotic glove for combined assistance and at-home rehabilitation, Robotics and Autonomous Systems 73, 2015, pp. 135-143, DOI: 10.1016/j.robot.2014.08.014.

[9] Ranzani T., Cianchetti M., Gerboni G., De Falco I., Menciassi A., A soft modular manipulator for minimally invasive surgery: design and characterization of a single module, IEEE Transactions on Robotics 32(1), 2016, pp. 187-200, DOI: 10.1109/TRO.2015.2507160.

[10] Payne C.J., Wamala I., Bautista-Salinas D., Saeed M., Van Story D., Thalhofer T., Horvath M.A., Aван C., Del Nido P.J., Walsh C.J., Vasilyev N.V., Soft robotic ventricular assist device with septal bracing for therapy of heart failure, Science Robotics 2(12), 2017, DOI: 10.1126/scirobotics.aan6736.

[11] Nguyen T.D., Han H.S., Shin H.Y., Nguyen C.T., Phung H., Hoang H.V., Choi H.R., Highly sensitive flexible proximity tactile array sensor by using carbon micro coils, Sensors and Actuators A: Physical 266, 2017, pp. 166-177, DOI: 10.1016/j.sna.2017.09.013.

[12] Ozel S., Keskin N.A., Khea D., Onal C.D., A precise embedded curvature sensor module for soft -bodied robots, Sensors and Actuators A: Physical 236, 2015, pp. 349-356, DOI: 10.1016/j.sna. 2015.09.041.

[13] Wang H., Zhang R., Chen W., Liang X., Pfeifer R., Shape detection algorithm for soft manipulator based on fiber Bragg gratings, IEEE/ASME Transactions on Mechatronics 21(6), 2016, pp. 2977 -2981, DOI: 10.1109/TMECH.2016.2606491.

[14] Monzon-Hernandez D., Martinez-Rios A., Torres-Gomez I., Salceda-Delgado G., Compact optical fiber curvature sensor based on concatenating two tapers, Optics Letters 36(22), 2011, pp. 4380 -4382, DOI: 10.1364/OL.36.004380.

[15] Zhou W., Zhou Y., Dong X., Shao L.Y., Cheng J., Albert J., Fiber-optic curvature sensor based on cladding-mode Bragg grating excited by fiber multimode interferometer, IEEE Photonics Journal 4(3), 2012, pp. 1051-1057, DOI: 10.1109/JPHOT.2012.2202895.

[16] He Y., Zhang X., Zhu L., Sun G., Lou X., Dong M., Optical fiber sensor performance evaluation in soft polyimide film with different thickness ratio, Sensors 19(4), 2019, article 790, DOI: 10.3390 / $\underline{\mathrm{s} 19040790 .}$.

[17] Sun G., Li H., Dong M., Lou X., Zhu L., Optical fiber shape sensing of polyimide skin for a flexible morphing wing, Applied Optics 56(33), 2017, pp. 9325-9332, DOI: 10.1364/AO.56.009325.

[18] He Y., Dong M., Sun G., Meng F., Song Y., Zhu L., Shape monitoring of morphing wing using micro optical sensors with different embedded depth, Optical Fiber Technology 48, 2019, pp. 179-185, DOI: 10.1016/j.yofte.2018.12.025.

[19] He Y., Zhu L., Sun G., Meng F., Song Y., Fiber Brag grating monitoring of a morphing wing based on a polyvinyl chloride reinforced silicone substrate, Optical Fiber Technology 50, 2019, pp. 145-153, DOI: 10.1016/j.yofte.2019.03.019.

[20] Zhao H., Jalving J., Huang R., Knepper R., Ruina A., Shepherd R., A helping hand: soft orthosis with integrated optical strain sensors and EMG control, IEEE Robotics \& Automation Magazine 23(3), 2016, pp. 55-64, DOI: 10.1109/MRA.2016.2582216.

[21] Xu R., Yurkewich A., PAtel R.V., Curvature, torsion, and force sensing in continuum robots using helically wrapped FBG sensors, IEEE Robotics and Automation Letters 1(2), 2016, pp. 1052-1059, DOI: $10.1109 /$ LRA.2016.2530867.

[22] He Y., Zhu L., Sun G., Yu M., Dong M., Design, measurement and shape reconstruction of soft surgical actuator based on fiber Bragg gratings, Applied Sciences 8(10), 2018, article 1773, DOI: 10.3390/app8101773. 
[23] Searle T.C., Althoefer K., Seneviratne L., Liu H., An optical curvature sensor for flexible manipulators, [In] 2013 IEEE International Conference on Robotics and Automation, 2013, pp. 4415 -4420, DOI: 10.1109/ICRA.2013.6631203.

[24] Silva A., Catarino A., Correia M., Frazao O., Design and characterization of a wearable macrobending fiber optic sensor for human joint angle determination, Optical Engineering 52(12), 2013, article 126106, DOI: 10.1117/1.OE.52.12.126106.

[25] Ge J., Feng H., Chen Y., Tse Z.T.H., FoK M.P., Spiral-structured fiber Bragg grating for contact force sensing through direct power measurement, Optics Express 22(9), 2014, pp. 10439-10445, DOI: $10.1364 /$ OE.22.010439.

[26] Ge J., JAMES A.E., Xu L., CHEN Y., Kwoк K.W., FoK M P., Bidirectional soft silicone curvature sensor based on off-centered embedded fiber Bragg grating, IEEE Photonics Technology Letters 28(20), 2016, pp. 2237-2240, DOI: 10.1109/LPT.2016.2590984.

[27] Sareh S., Noh Y., Li M., Ranzani T., Liu H., Althoefer K., Macrobend optical sensing for pose measurement in soft robot arms, Smart Materials and Structures 24(12), 2015, article 125024, DOI: 10.1088/0964-1726/24/12/125024.

[28] Xu L., Liu N., Ge J., WAng X., FoK M.P., Stretchable fiber-Bragg-grating-based sensor, Optics Letters 43(11), 2018, pp. 2503-2506, DOI: 10.1364/OL.43.002503.

[29] Rus D., Tolley M.T., Design, fabrication and control of soft robots, Nature 521(7553), 2015, pp. 467 -475 , DOI: 10.1038 /nature14543.

[30] Connolly F., Walsh C.J., Bertoldi K., Automatic design of fiber-reinforced soft actuators for trajectory matching, Proceedings of the National Academy of Sciences of the United States of America 114(1), 2017, pp. 51-56, DOI: 10.1073/pnas.1615140114.

Received September 29, 2020 in revised form December 1, 2020 